\title{
Ontology-Based Search of Genomic Metadata
}

\author{
Javier D. Fernández, Maurizio Lenzerini \\ Dipartimento di Ingegneria Informatica Automatica e Gestionale Antonio Ruberti, \\ Sapienza Università di Roma, Italy \\ Email: $\{$ fernandez,lenzerini\}@dis.uniroma1.it \\ Marco Masseroli, Francesco Venco, Stefano Ceri \\ Dipartimento di Elettronica, Informazione e Bioingegneria, Politecnico di Milano, Italy \\ Email: $\{$ stefano.ceri,marco.masseroli,francesco.venco\}@polimi.it
}

\begin{abstract}
The Encyclopedia of DNA Elements (ENCODE) is a huge and still expanding public repository of more than 4,000 experiments and 25,000 data files, assembled by a large international consortium since 2007; unknown biological knowledge can be extracted from these huge and largely unexplored data, leading to data-driven genomic, transcriptomic and epigenomic discoveries. Yet, search of relevant datasets for knowledge discovery is limitedly supported: metadata describing ENCODE datasets are quite simple and incomplete, and not described by a coherent underlying ontology. Here, we show how to overcome this limitation, by adopting an ENCODE metadata searching approach which uses high-quality ontological knowledge and state-of-the-art indexing technologies. Specifically, we developed S.O.S. GeM (http://www.bioinformatics.deib.polimi.it/SOSGeM/), a system supporting effective semantic search and retrieval of ENCODE datasets. First, we constructed a Semantic Knowledge Base by starting with concepts extracted from ENCODE metadata, matched to and expanded on biomedical ontologies integrated in the well-established Unified Medical Language System; we prove that this inference method is sound and complete. Then, we leveraged the Semantic Knowledge Base to semantically search ENCODE data from arbitrary biologists' queries; this allows correctly finding more datasets than those extracted by a purely syntactic search, as supported by the other available systems. We empirically show the relevance of found datasets to the biologists' queries.
\end{abstract}

Index Terms-Semantic search, Genomic data retrieval, Genomic data and knowledge management, Encyclopedia of DNA Elements

\section{INTRODUCTION}

Continuous improvements of Next Generation Sequencing (NGS) technologies in quality, cost of results ${ }^{1}$ and sequencing time are leading shortly to the possibility of sequencing an entire human genome in few minutes for a cost of less than $\$ 1,000$ [1], [2]. As a consequence, very largescale sequencing projects are emerging, including the 1000 Genomes Project, aiming at establishing an extensive catalog of human genomic variation [3], The Cancer Genome Atlas (TCGA), a full-scale effort to explore the entire spectrum of genomic changes involved in human cancer [4], and the Encyclopedia of DNA elements (ENCODE) [5].

The ENCODE project is the most general and relevant world-wide repository fueling basic biology research. It provides public access to more than 4,000 experimental datasets, including the just released data from its Phase 3, which comprise hundreds of experiments of mainly RNAseq, ChIP-seq and DNase-seq assays in human and mouse. The high availability of many different genomic features in distinct conditions and of a new generation of bioinformatics systems [6], [7] enables the discovery of genetic and epigenetic phenomena, offering huge opportunities for a variety of applications (notably cancer research).

But availability of ENCODE datasets is not effective in the lack of adequate search systems. Unfortunately, while the quality of experimental data is typically very high, the

1. http://www.genome.gov/sequencingcosts/ documentation and associated metadata is not comparatively rich or equally curated, resulting in a difficulty to locate all the experimental data corresponding to given phenomena. Current interfaces to ENCODE data, available from both the UCSC Genome Bioinformatics site ${ }^{2}$ and ENCODE Project Portal $^{3}$, provide very useful exploration, browsing, visualization and downloading functionalities, but partial support for metadata extraction and only limited search capabilities; to date, the evaluation of an ENCODE data search query is strictly based only on syntactic (textual) matching of search terms. A state-of-the-art "syntactic-based" retrieval system allows to retrieve a set of similar results (e.g. on the basis of well-known technologies such as Apache Lucene $^{4}$ ); yet, this functionality is again based only on string distances. This prevents finding items described with synonyms or semantic variants of the query terms used. Additional support for such advanced search capabilities is needed in order to significantly increase the number and quality of relevant datasets found.

In this work, we overcome current limitations in the search for ENCODE datasets by supporting ontology-based search of their metadata. For our genomic and semantic purposes, we consider the global ontology provided by the

2. http://genome.ucsc.edu/ENCODE/

3. http://www.encodeproject.org/

4. https://lucene.apache.org/ 
Unified Medical Language System (UMLS) [8], which collects and integrates well-established biomedical ontologies.

Intuitively, our approach relies on semantically annotating the metadata about ENCODE datasets by means of UMLS, and completing the information by materializing inferred facts, i.e. by performing the semantic closure [9] of such annotations. Then, we set up an abstraction layer to allow users searching relevant ENCODE experiments from text-based queries.

This approach has been practically implemented in S.O.S. GeM${ }^{5}$, standing for Sapienza Ontology-based Search of Genomic Metadata. Thus, the S.O.S. GeM system builds a Semantic Knowledge Base (SKB) of ENCODE metadata that includes the concepts extracted from ENCODE experiment metadata and their associated concepts inferred by using the UMLS global ontology. Then, S.O.S. GeM provides an intuitive Web-based interface in which users are simply asked for a text query. The proposed query answering algorithm inspects the user query for both UMLS concepts and interesting syntactic tokens, obtaining the relevant set of ENCODE metadata from the SKB and providing access to the related ENCODE datasets.

S.O.S GeM is part of a larger project called GenData $\mathbf{2 0 2 0}^{6}$, which has recently produced a high-level, declarative GenoMetric Query Language (GMQL) ${ }^{7}$ [7] for querying heterogeneous NGS data. In other words, the user query answers semantically computed by S.O.S. GeM can be directly routed to the GMQL query processing engine, serving an integrated semantic access for fine-grained genomic queries.

This paper is structured as follows. Section 2 provides some background on the GenData 2020 project, and Section 3 presents the state of the art. Then, Section 4 dwells into the description of our proposed solution, discussing the ontology construction, then the query formulation and the query processing algorithm, including the proof of soundness and completeness. Section 5 illustrates the system implementation, and Section 6 presents the system evaluation both in terms of size of involved data and of offline and online performance; Section 7 concludes.

\section{Background: The Gendata 2020 Project}

GenData $2020^{6}$ is a large project sponsored by the Italian Ministry of University and Research, advocating a new, holistic approach to genomic data management that uses cloud-based computing. Data are organized as datasets consisting of several data samples, each containing many genomic regions, where each sample is associated with different experimental conditions described by its metadata; one can think to samples as objects and to datasets as their containers. Our objective is not to address raw data processing, but rather to embrace all processed data formats through an interoperable data model, and by enabling queries over tens of datasets, hundreds or even thousands of data samples and several millions of genomic regions thereby opening genomics to big data management.

5. http:/ / www.bioinformatics.deib.polimi.it/SOSGeM/

6. http://www.bioinformatics.deib.polimi.it/GenData/

7. http://www.bioinformatics.deib.polimi.it/GMQL/
GenData 2020 has adopted a new data model, called Genomic Data Model (GDM) [7], providing two fundamental abstractions for each data sample:

- Metadata describe the biological and clinical properties associated with each sample, e.g. experimental condition, cell line, biological sample, antibody used in experiment preparation, considered antibody target, and also patient phenotype when data have clinical nature. Due to the great heterogeneity of the metadata information that can be associated with each sample, they are represented as arbitrary attribute-value pairs.

- A region corresponds to all the DNA nucleotides whose position is between the region left and right ends, typically within a chromosome; in general, we do not include a full nucleotide sequence within the region data, but rather we store high-level properties of the region, which are produced by the post-processing of sequencing data.

Gendata 2020 has also defined and implemented a new, high-level query language for bio-informaticians, called GenoMetric Query Language (GMQL) ${ }^{7}$ [7], which enables building new datasets from a repository of existing datasets. S.O.S. GeM can be used as the first component of GMQL query execution workflow, by producing an enhanced set of ENCODE experiments corresponding to the query conditions; of course, it can also be used stand-alone ${ }^{5}$.

\section{State of the ART}

In the last decade, semantic developments and biology research are following intersecting paths. A nice overview on big biological databases, bio-ontologies and knowledge discovery problems can be found in [10], [11], [12], [13]. In particular, ontology-based access to biological repositories is a relevant and challenging area. The TAMBIS architecture [14] was one of the pioneer projects addressing the challenging issue of integrating and querying different bioinformatics sources through a model of domain knowledge in a transparent way to the users. In [15], Xuan et al. proposed an ontology-based exploratory system, called PubOnto, to enable the interactive exploration and filtering of search results in the medical publication database Medline, using multiple ontologies taken from the well-established Open Biological and Biomedical Ontologies (OBO) foundry ${ }^{8}$. The authors developed a general purpose ontology to free-text mapping which relies on the pre-generation of lexical variations, word order permutations of ontology terms, their synonyms and a suffix-tree based string matching algorithm.

The Gene Ontology (GO) project [16], founded in 1998, is a notable collaborative effort to address the need for consistent descriptions of gene products. The GO project has developed three ontologies that describe gene products in terms of their associated biological processes, cellular components and molecular functions in a species-independent manner. An interesting application that makes use of the GO is GoPubMed [17]. It is a service that submits keywords to the PubMed repository of medical publication abstracts, extracts GO terms from the retrieved abstracts and presents the induced ontology for browsing; such ontology is the

8. http://www.obofoundry.org/ 
minimal GO subset which comprises all the GO terms found in the retrieved documents.

A similar approach was developed by Müller et al. in Textpresso [18], a text-mining system for scientific literature. It splits papers into sentences and sentences into words or phrases. Each word or phrase is then labeled according to the lexicon of the Textpresso ontology, mainly built from GO. Sentences are indexed with respect to labels and words to allow a rapid search for sentences that have a desired label and/or keyword.

The Conceptual Open Hypermedia Service (COHSE) by Bechhofer et al. [19] constructs hypertext structures using the information that an ontology encodes. The COHSE Agent takes documents/pages in and enhances them with links derived through an ontology and associated lexicon along with a mapping from concepts to possible link targets.

Several works explore the query expansion strategy in the medical domain to improve the precision and recall of queries. Zhu et al. [20], and more recently Thesprasith and Jaruskulchai [21], first identify medical terms in the query using a basic lexical tool and match them to MeSH ontology concepts. Then, they expand the found concepts by adding UMLS co-concepts, i.e. semantic terms that often appears together. In order to restrict the query expansion, they rank expanded terms by frequency and apply some thresholds. Díaz-Galiano et al. [22] performed a simpler approach and used MeSH descriptors to expand the queries on a collection composed of images and text. All these approaches, though, report slight improvements and are applied to limited domains and ontologies, which may show scalability issues at large scale once term expansion largely increases. A review on ontology-based query expansion can be found in [23].

Complementary works focus on ontology-based automatic annotation. In [24], Taboada et al. address semantic annotation of relevant literature about rare disease patients. They identify concepts by means of the entity recognizer Mgrep [25] (a comparison between Mgrep and MetaMap is performed in [26]) and expand them using the hierarchical structure of their considered $\mathrm{OBO}$ ontologies. Jonquet $e t$ al. [27] apply a similar approach to first annotate biomedical resources (patient records, academic articles, etc.) with the concepts in the National Center for Biomedical Ontology (NCBO). Then, analogously to the previous work, they recognize the concepts in user queries with Mgrep and expand them with NCBO hierarchies. However, it is not possible to test the correctness of the latter approaches as neither details on the closure nor a formal semantics are given.

\section{Ontology-Based Search: Description of OUR SOLUTION}

We base the task of defining an ontology to support the search for genomic datasets on two important items that we assume available:

- A set $M$ of metadata about a collection of genomic experiments and samples (or data files). In particular, for a sample $S$ of an experiment and for a metadata attribute of the collection, $M$ specifies which is the value associated with $S$ by the attribute.

- An ontology $\mathcal{G}$, called the global ontology, which is the union of all the ontologies that are considered relevant for describing the domain of genomics, and are accessible through UMLS.

Let $(\mathrm{A}, v)$ be the metadata pair associated with a sample $S$ in $M$, specifying that $v$ is the value associated with $S$ by the attribute A. From such value $v$, which can always be seen as a string, the following data are extracted:

- a set $t_{S}^{\mathrm{A}}(M)$ of strings, called tokens, representing relevant substrings of the string $v$;

- a set of classes $c_{S}^{\AA}(M)$ that are present in the global ontology, representing relevant classes that, either implicitly or explicitly, are mentioned in $v$, according to a text analysis of $v$.

We will make use of the above two notions in the following, where the mechanism to extract $t_{S}^{\mathrm{A}}(M)$ and $c_{S}^{\mathrm{A}}(M)$ for the various A and $S$ are described.

Given the metadata set $M$ and the global ontology $\mathcal{G}$, we build the ontology $\mathcal{O}_{\mathcal{M}, \mathcal{G}}$ on the basis of $M$ and $\mathcal{G}$. When $M$ and $\mathcal{G}$ are understood, we simplify the notation and denote the ontology by $\mathcal{O}$. To specify $\mathcal{O}$, we use the ontology language OWL $2 \mathrm{QL}$, a profile of OWL 2 derived from the DL-lite family [28], specifically designed to perform ontology-based accesses to big data collections while keeping inferences tractable. We refer the reader to [29] for a complete description of OWL 2 QL. Here, we only recall some of the most important notions. A class (or concept) is a unary predicate representing a set of individual objects (or simply individuals) in the domain of interest, called its instances. A data property is a binary predicate representing an attribute, i.e. a relation associating with the individuals a set of values of a particular type (i.e. a string). An object property is a binary predicate representing a relationship between classes, i.e. between the instances of such classes.

Given an alphabet $\Sigma$ for individuals, classes, data properties and object properties, an ontology in OWL 2 QL is a set of OWL 2 QL axioms, where each axiom is a formula over the alphabet $\Sigma$. Here are types and intuitive semantics of OWL 2 QL axioms of particular importance for our task:

- Subclassof ( $C D$ ), stating that all instances of class $C$ are also instances of class $D$;

- SubobjectPropertyof ( $R Q$ ), stating that all pairs of objects that are instances of the object property $R$ are also instances of the object property $Q$;

- SubDataPropertyof $\left(A_{1} A_{2}\right)$, stating that all pairs that are instances of the data property $A_{1}$ are also instances of the data property $A_{2}$;

- ObjectPropertyDomain ( $R C$ ), stating that, in all the pairs that are instances of the object property $R$, the first component of the pair is an instance of the class $C$;

- ObjectPropertyRange ( $R C$ ), stating that, in all the pairs that are instances of the object property $R$, the second component of the pair is an instance of the class $C$;

- DataPropertyDomain ( $A C$ ), stating that, in all the pairs that are instances of the data property $A$, the first component of the pair is an instance of the class $C$;

- ClassAssertion ( $C a$ ), stating that $a$ is an instance of class $C$;

- ClassAssertion ( ObjectSomeProperty ( $R$ $C$ ) $a$ ), stating that there is an object $x$ such that $(i)$ 
$(a, x)$ is an instance of the object property $R$, and $(i i) x$ is an instance of $C$;

- ObjectPropertyAssertion ( $R a b)$, stating that $(a, b)$ is an instance of the object property $R$;

- DatapropertyAssertion ( $A a s)$, stating that $(a, s)$ is an instance of the data property $A$.

The formal semantics of OWL 2, and therefore of OWL $2 \mathrm{QL}$, is based on the classical notion of interpretation in logic. An interpretation $\mathcal{I}$ for an ontology defined over the alphabet $\Sigma$ is a pair $\left(\Delta^{\mathcal{I}},{ }^{\mathcal{I}}\right)$, where $\Delta^{\mathcal{I}}$ is a non-empty set, called the domain of $\mathcal{I}$, and ${ }^{\mathcal{I}}$ is the interpretation function of $\mathcal{I}$, i.e. a function assigning an element of $\Delta^{\mathcal{I}}$ to every constant in $\Sigma$, a subset of $\Delta^{\mathcal{I}}$ to every class in $\Sigma$, a set of pairs of elements in $\Sigma$ for every data property and every object property in $\Sigma$. We now specify when an interpretation $\mathcal{I}$ satisfies an axiom $\alpha$, written $\mathcal{I} \models \alpha$.

- $\mathcal{I}=$ SubClassof $(C D)$ if $C^{\mathcal{I}} \subseteq D^{\mathcal{I}}$.

- $\mathcal{I}=$ SubobjectProperty $(R Q)$ if $R^{\mathcal{I}} \subseteq Q^{\mathcal{I}}$.

- $\mathcal{I}=$ SubDataPropertyof $\left(A_{1} A_{2}\right)$ if $A_{1}^{\mathcal{I}} \subseteq A_{2}^{\mathcal{I}}$.

- $\mathcal{I}=$ objectPropertyDomain $(R C)$ if for all $(a, b) \in R^{\mathcal{I}}$, we have that $a \in C^{\mathcal{I}}$.

- $\mathcal{I}=$ objectPropertyRange $(R C)$ if for all $(a, b) \in R^{\mathcal{I}}$, we have that $b \in C^{\mathcal{I}}$.

- $\mathcal{I} \models$ DatapropertyDomain $(A C)$ if for all $(a, s) \in R^{\mathcal{I}}$, we have that $a \in C^{\mathcal{I}}$.

- $\mathcal{I}=$ ClassAssertion $(C a)$ if $a^{\mathcal{I}} \in C^{\mathcal{I}}$.

- $\mathcal{I}=$ ClassAssertion ( ObjectSomeProperty ( $R C$ ) $a)$ if there exists $b \in \Delta^{\mathcal{I}}$ such that $\left(a^{\mathcal{I}}, b\right) \in$ $R^{\mathcal{I}}$, and $b \in C^{\mathcal{I}}$.

- $\mathcal{I} \equiv$ ObjectPropertyAssertion $(R a b)$ if $\left(a^{\mathcal{I}}, b^{\mathcal{I}}\right) \in R^{\mathcal{I}}$.

- I $=$ DataPropertyAssertion ( $A a s$ ) if $\left(a^{\mathcal{I}}, s^{\mathcal{I}}\right) \in A^{\mathcal{I}}$.

As usual, we say that $\mathcal{I}$ is a model of $\mathcal{O}$, written $\mathcal{I}=\mathcal{O}$, if all the axioms of $\mathcal{O}$ are satisfied by $\mathcal{I}$, and we say that an axiom $\alpha$ is logically implied by an ontology $\mathcal{O}$, written $\mathcal{O}=\alpha$, if $\alpha$ is satisfied by every model of $\mathcal{O}$.

\subsection{The Ontology}

We now turn our attention to the issue of how to build the ontology $\mathcal{O}$ in our setting. As we said before, we base the task of defining $\mathcal{O}$ on the metadata $M$ and the global ontology $\mathcal{G}$. We first specify the alphabet of $\mathcal{O}$ and then we illustrate its axioms.

The alphabet of $\mathcal{O}$ is constituted by the set $\Sigma_{\mathcal{G}}$ of class symbols that are present in the global ontology $\mathcal{G}$, plus another set of symbols, disjoint from $\Sigma_{\mathcal{G}}$, comprising:

- the individual object symbols, at least one for each experiment and one for each sample represented in $M$,

- the constants of type string, at least one for each token present in $M$,

- the class symbols Experiment, representing all experiments managed by the knowledge base, and Sample, representing the samples associated with the experiments,

- the object property symbol consistsof, which associates to each experiment the corresponding samples,

- the data property symbols experimentTypeand dccAccession, which provide, for each instance of
Experiment, its type and a value identifying such instance, respectively,

- the data property symbol hasValueFor-A, for each attribute $\mathrm{A}$ that is present in the metadata set $M$, representing the association between $S$ and the tokens in $t_{S}^{\mathrm{A}}(M)$,

- the object property symbol hasLinkTo-A, for each attribute $\mathrm{A}$ that is present in the metadata set $M$, which represents the association between $S$ and a set of objects that are instances of the various classes in $c_{S}^{\mathrm{A}}(M)$,

- the data property symbol hasAssociatedValue,

- the object property symbol hasAssociatedObject.

Note that, regarding hasLinkTo-A, we do not have an exact knowledge about the objects that are instances of classes in $c_{S}^{\mathrm{A}}(M)$. Therefore, we model them using the notion of existential quantification in logic, and more precisely in OWL 2 QL. In particular, if C is a class in $c_{S}^{\mathrm{A}}(M)$, then we sanction that there is some object $B$ such that the pair $(S, B)$ is an instance of the relationship hasLinkTo-A, and $B$ is an instance of $\mathrm{C}$. A graphical representation of the ontology $\mathcal{O}$ appears in Figure 1.

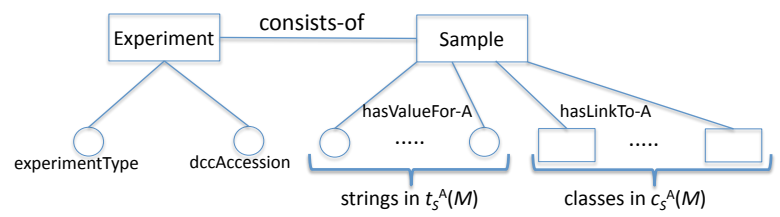

Fig. 1. A graphical representation of the ontology $\mathcal{O}$.

Taking into account the above intuitive considerations, we now provide the precise definition of the axioms of the ontology $\mathcal{O}$ on the basis of $M$ and $\mathcal{G}$, as follows.

- All the axioms of $\mathcal{G}$ are in $\mathcal{O}$; we observe that all the axioms of this category are of the form SubclassOf $C D$ ).

- For every experiment $E$ in $M$, the axiom ClassAssertion (Experiment $e$ ) is in $\mathcal{O}$, where e is the individual representing $E$.

- For every sample $S$ associated with the experiment $E$ in $M$, the axioms ClassAssertion ( Sample s ), ObjectPropertyAssertion ( consistsof e s ) are in $\mathcal{O}$, where $s$ and $e$ are the individuals representing $S$ and $E$, respectively.

- The axioms ObjectPropertyDomain ( consistsof Experiment), and ObjectPropertyRange ( consistsof Sample ) are in $\mathcal{O}$, stating that the domain and the range of the relation consistsof are Experiment and Sample, respectively.

- For every data property hasValueFor-A, the axiom SubDataPropertyof ( hasValueFor-A hasAssociatedValue) is in $\mathcal{O}$, stating that hasValueFor-A is a subset of the data property hasAssociatedValue.

- The axioms DataPropertyDomain ( hasAssociatedValue Sample) is in $\mathcal{O}$, stating that the data property hasAssociatedValue is defined on the class Sample. Note that this implies that every data property hasValueFor-A is also defined on the class Sample. 
- For every object property hasLinkTo-A, the axiom SubObjectPropertyof ( hasLinkToA hasAssociatedobject) is in $\mathcal{O}$, stating that hasLinkTo-A is a subset of the object property hasAssociatedobject.

- The axioms ObjectPropertyDomain ( hasAssociatedobject Sample ) is in $\mathcal{O}$, stating that the object property hasAssociatedobject is defined on the class Sample. Note that this implies that every object property hasLinkTo-A is also defined on the class Sample.

- For every data property hasValueFor-A, for every $s$ asserted to be an instance of sample in $\mathcal{O}$, and for every value $\mathrm{v}$ in $t_{\mathrm{s}}^{\text {hasvaluefor-A }}(M)$, the axiom DataPropertyAssertion ( hasValueFor-A $\mathrm{S} \mathrm{v}$ ) is in $\mathcal{O}$.

- For every object property hasLinkTo-A, for every $\mathrm{s}$ asserted to be an instance of sample in $\mathcal{O}$, and for every class $\mathrm{C}$ in $\mathrm{C}_{\mathrm{s}}^{\text {hasLinkTo-A }}(M)$, the axiom ClassAssertion ( objectSomeProperty ( hasLinkTo-A C ) $s$ ) is in $\mathcal{O}$.

\subsection{Queries}

We now define the class of queries that we are interested in, which we call search queries. We express search queries in SPARQL, and we obviously assume the OWL 2 Direct Semantics entailment regime for such queries. Actually, we first define a subclass of search queries, the subclass of positive search queries. Intuitively, a query of this class asks for all samples that are related, by means of any attribute in their metadata, to a given set of tokens and to a given set of classes. Formally, given tokens $v_{1}, \ldots, v_{m}$ and classes $C_{1}, \ldots, C_{n}$ that we want to be related to the samples we are searching for, the corresponding positive search SPARQL query is defined as:

$$
\begin{aligned}
& \text { select } ? x \\
& \text { where }\left\{\alpha_{1} \cdot \cdots \cdot \alpha_{m} \cdot \beta_{1} \cdot \gamma_{1} \cdots \cdot \beta_{n} \cdot \gamma_{n}\right\}
\end{aligned}
$$

where

- $m \geq 0, n \geq 0$, and $m+n>0$,

- the block following the word where is called the body of the positive search query, and is constituted simply by a conjunction of atoms (in SPARQL, the atoms in a conjunction are separated by "."),

$$
\begin{aligned}
& \text { - each } \alpha_{i} \text { is an atom of the } \\
& \text { form } \\
& \text { hasAssociatedvalue ? } x v_{i} \text { ), } \\
& \text { - each } \beta_{i} \text { is an atom of the } \\
& \text { form objectPropertyAssertion ( } \\
& \text { hasAssociatedobject ? ? } y_{i} \text { ), } \\
& \text { - each } \gamma_{i} \text { is an atom of the form ClassAssertion ( } C \\
& \text { ? } \left.y_{i}\right) \text {. }
\end{aligned}
$$

The intuitive semantics of the above query in an interpretation $\mathcal{I}$ for $\mathcal{O}$ is as follows: the query asks for all samples that in $\mathcal{I}$ are related to the various input tokens $v_{1}, \ldots, v_{m}$ by means of the data property hasAssociatedValue, and are related by means of the object property hasAssociatedobject to at least one individual (represented by ? $y_{i}$ ) that is an instance of $C_{i}$, for each $C_{i}$ in the set of the input classes $C_{1}, \ldots, C_{n}$.

Formally, if $Q$ is a positive search query of the above form, and $\mathcal{I}$ is an interpretation for $\mathcal{O}$, the extension of query $Q$ in $\mathcal{I}$, denoted by $Q^{\mathcal{I}}$, is defined as the set of individuals $d$ in $\mathcal{O}$ such that:

- for each $\alpha_{i}$ in the body of $Q, \quad\left(d, v_{i}\right) \in$ hasAssociatedValue ${ }^{\mathcal{I}}$,

- for each $\beta_{i} \cdot \gamma_{i}$ in the body of $Q$, there is some $b \in C_{i}^{\mathcal{I}}$ such that $(\mathrm{d}, b) \in$ hasAssociatedob ject ${ }^{\mathcal{I}}$.

With the notion of extension of a query in an interpretation in place, we can now provide the definition of certain answer to a query $Q$ with respect to an ontology $\mathcal{O}$. An individual $\mathrm{d}$ is a certain answer to $Q$ with respect to the ontology $\mathcal{O}$, if $\mathrm{d} \in Q^{\mathcal{I}}$ for every model $\mathcal{I}$ of $\mathcal{O}$. Finally, the set of certain answers to $Q$ with respect to $\mathcal{O}$, denoted as $\operatorname{cert}_{\mathcal{O}}(Q)$, is simply the set constituted by each individual that is a certain answer to $Q$ with respect to $\mathcal{O}$, i.e. $\operatorname{cert}_{\mathcal{O}}(Q)=\bigcap_{\mathcal{I} \in\{\mathcal{J} \mid \mathcal{J} \models \mathcal{O}\}} Q^{\mathcal{I}}$.

We now turn to the class of basic search queries. Informally, a query of this class simply asks for the difference between two positive search queries. Given tokens $v_{1}, \ldots, v_{m}$ and classes $C_{1}, \ldots, C_{n}$ representing the features we want the desired samples to have, and given tokens $v_{1}^{\prime}, \ldots, v_{m}^{\prime}$ and classes $C_{1}^{\prime}, \ldots, C_{n}^{\prime}$ representing the features that we do not want the desired samples to have, the corresponding basic search query is defined as follows:

$$
\begin{aligned}
& \text { select ? } x \\
& \text { where }\left\{\left\{\alpha_{1} \cdots \cdot \alpha_{m} \cdot \beta_{1} \cdot \gamma_{1} \cdots, \beta_{n} \cdot \gamma_{n}\right\}\right. \\
& \text { MINUS } \\
& \left\{\left\{\alpha_{1}^{\prime}\right\} \text { UNION } \cdots \text { UNION }\left\{\alpha_{m^{\prime}}^{\prime}\right\}\right. \text { UNION } \\
& \left.\left\{\beta_{1}^{\prime} \cdot \gamma_{1}^{\prime}\right\} \text { UNION } \cdots \text { UNION }\left\{\beta_{n^{\prime}}^{\prime} \cdot \gamma_{n^{\prime}}^{\prime}\right\}\right\}
\end{aligned}
$$

where

- $m \geq 0, n \geq 0, m+n>0, m^{\prime} \geq 0$, and $n^{\prime} \geq 0$.

- As usual, the (complex) block following the word where is called the body of the query.

- The block following the reserved word MINUS is called the MINUS-block of the query.

- If $m^{\prime}=0$ and $n^{\prime}=0$, then the MINUS-block is missing.

- The select query with ? $x$ as a distinguished variable and $\left\{\alpha_{1}, \cdots . \alpha_{m} . \beta_{1} \cdot \gamma_{1} \cdots . \beta_{n} \cdot \gamma_{n}\right\}$ as a body, i.e. the query

select ? $x$

where $\left\{\alpha_{1}, \cdots . \alpha_{m} \cdot \beta_{1} \cdot \gamma_{1} \cdots \cdot \beta_{n} \cdot \gamma_{n}\right\}$

is called the positive part of $Q$ and is denoted $Q_{p}$.

- The select query with ? $x$ as a distinguished variable and the MINUS-block as body, i.e. the query

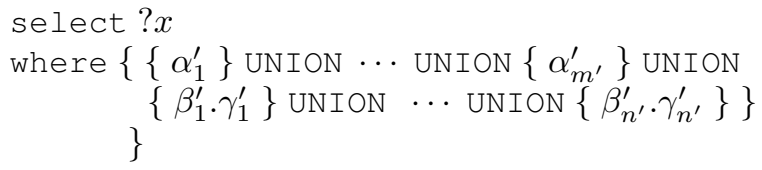

is called the negative part of $Q$ and is denoted $Q_{n}$. Note that the body of the negative part is constituted by the union of several atoms, where the semantics of union is the usual one in SPARQL (the result of the union query is the union of the results of the components).

The intuitive semantics of a basic search query $Q$ is the obvious one: $Q$ asks for all the samples that are among 
the certain answers to the positive part $Q_{p}$ of $Q$, but are not among the certain answers to the negative part $Q_{n}$ of $Q$. Thus, the formal semantics of basic search queries is immediate: given an ontology $\mathcal{O}$ and a basic search query $Q$, we have that $\operatorname{cert}_{\mathcal{O}}(Q)=\operatorname{cert}_{\mathcal{O}}\left(Q_{p}\right) \backslash \operatorname{cert}_{\mathcal{O}}\left(Q_{n}\right)$.

Finally, we introduce the notion of search query. In our approach a search query is simply the union of a set of basic search queries. More precisely, if $Q_{1}, \ldots, Q_{h}$ are basic search queries, then the search query built on them is the query of the form:

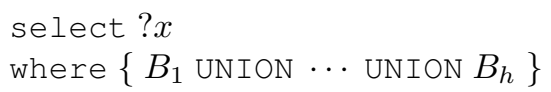

where $B_{1}, \ldots, B_{h}$ are the bodies of $Q_{1}, \ldots, Q_{h}$, respectively. The formal semantics of search queries is as follows: given an ontology $\mathcal{O}$ and a search query $Q$ built on $Q_{1}, \ldots, Q_{h}$, we have that $\operatorname{cert}_{\mathcal{O}}(Q)=\operatorname{cert}_{\mathcal{O}}\left(Q_{1}\right) \cup \cdots \cup \operatorname{cert}_{\mathcal{O}}\left(Q_{h}\right)$.

\subsection{The Query Answering Algorithm}

In principle, there are two approaches for computing the set of certain answers of a search query $Q$ with respect to an ontology $\mathcal{O}$. The first approach is based on query rewriting [28]: taking into account the intensional axioms of the ontology $\mathcal{O}$, the query is rewritten into a new query $Q^{\prime}$, which is evaluated over the extensional portion of the ontology, seen as a database. Unfortunately, this approach is not suited to our scenario, because, due to the size of $\mathcal{G}$, the size of the rewritten query may be prohibitive. The second approach is based on materialization [9]: the ontology $\mathcal{O}$ is transformed into another ontology $\mathcal{O}^{\prime}$ obtained from $\mathcal{O}$ by adding suitable extensional axioms implied by $\mathcal{O}$ itself, and then the query $Q$ is evaluated over the ontology $\mathcal{O}^{\prime}$ seen as a database. The latter approach is the one that we follow in our work. As such, our method is similar to the one presented in [30], developed in the context of Web search. However, both our notion of search query and our notion of materialization of an ontology (hereafter called completion) are specialized to genomic metadata, and therefore differ considerably from the one presented in [30]. In particular, in our context, the completion of the ontology includes axioms that are not taken into account in [30], requiring different proofs of the correctness of the method.

Before delving into the details of the algorithm, we need three new notions, namely the notions of evaluation of a search query over an ontology, completion of an ontology, and canonical model of the completion of an ontology.

We start with the evaluation of a search query $Q$ over an ontology $\mathcal{O}$, by first referring to positive search queries, and then generalizing to the whole class of search queries. If $Q$ is a positive search query of the form:

$$
\begin{aligned}
& \text { select } ? x \\
& \text { where }\left\{\alpha_{1} \cdot \cdots \cdot \alpha_{m} \cdot \beta_{1} \cdot \gamma_{1} \cdots \cdot \beta_{n} \cdot \gamma_{n}\right\}
\end{aligned}
$$

where each $\alpha_{i}$ has the form DataPropertyAssertion ( hasAssociatedValue ? $x v_{i}$ ), each $\beta_{i}$ has the form ObjectPropertyAssertion ( hasAssociatedobject $\left.? x ? y_{i}\right)$, and each $\gamma_{i}$ has the form ClassAssertion ( $\left.C ? y_{i}\right)$, then the evaluation of $Q$ over $\mathcal{O}$, denoted $\operatorname{eval}_{\mathcal{O}}(Q)$, is the set of individuals d in $\mathcal{O}$ such that:

- for each $\alpha_{i}$, the assertion DataPropertyAssertion ( hasAssociatedValue d $v_{i}$ ) is in $\mathcal{O}$, and

- for each $\beta_{i}$, the assertion ClassAssertion ( object SomeProperty ( hasAssociatedobject $C_{i}$ ) d ) is in $\mathcal{O}$.

If $Q$ is a basic search query, then the evaluation of $Q$ over $\mathcal{O}$ is defined as $\operatorname{eval}_{\mathcal{O}}\left(Q_{p}\right) \backslash \operatorname{eval}_{\mathcal{O}}\left(Q_{n}\right)$, where $Q_{p}$ is the positive part of $Q$, and $Q_{n}$ is the negative part of $Q$. Finally, if $Q$ is a search query built on queries $Q_{1}, \ldots, Q_{h}$, then the evaluation of $Q$ over $\mathcal{O}$ is defined as $\operatorname{eval}_{\mathcal{O}}\left(Q_{1}\right) \cup \cdots \cup \operatorname{eval}_{\mathcal{O}}\left(Q_{h}\right)$.

Note the difference between evaluating a query and computing its certain answers. When we evaluate a query over an ontology, we essentially treat the ontology assertions as a database, and we evaluate the query over such database. When we compute the certain answers to a query with respect to an ontology, we must consider all the models of the ontology, and return those tuples that are answers to the query in all such models.

We now define the notion of completion of an ontology $\mathcal{O}$. Roughly speaking, the completion $\mathcal{O}^{\prime}$ of $\mathcal{O}$ is obtained from $\mathcal{O}$ by adding all assertions on the instances of Sample that are logically implied by $\mathcal{O}$. More precisely, we obtain $\mathcal{O}^{\prime}$ by first letting all the axioms of $\mathcal{O}$ be also in $\mathcal{O}^{\prime}$, and then by repeating the following rules until no more axiom can be added:

- For every pair $(a, b)$ such that the axiom objectPropertyAssertion ( consistsof a

$\mathrm{b}$ ) is in $\mathcal{O}^{\prime}$, add the axiom ClassAssertion ( Sample b) and the axiom ClassAssertion ( Experiment a ) to $\mathcal{O}^{\prime}$, if they are not already in $\mathcal{O}^{\prime}$;

- For every pair $(a, b)$ such that the axiom DataPropertyAssertion ( hasValueFor-A a $\mathrm{V}$ ) is in $\mathcal{O}^{\prime}$, add the axiom DataPropertyAssertion ( hasAssociatedValue a $\mathrm{v}$ ) to $\mathcal{O}^{\prime}$, if it is not already in $\mathcal{O}^{\prime}$;

- For every pair $(\mathrm{a}, \mathrm{b})$ such that ObjectPropertyAssertion ( hasLinkTo-A a b ) is in $\mathcal{O}^{\prime}$, add the axiom ObjectPropertyAssertion ( hasAssociatedobject a b ) to $\mathcal{O}^{\prime}$, if it is not already in $\mathcal{O}^{\prime}$;

- For every assertion ClassAssertion ( ObjectSomeProperty ( hasAssociatedobject $C_{i}$ ) a ) in $\mathcal{O}^{\prime}$, and for every class $C_{j}$ such that $\mathcal{O}^{\prime}=$ subsetof $\left(C_{i} C_{j}\right)$, add the assertion ClassAssertion ( ObjectSomeProperty ( hasAssociatedobject $C_{j}$ ) a ) to $\mathcal{O}^{\prime}$, if it is not already in $\mathcal{O}^{\prime}$.

It is easy to see that $\mathcal{O}^{\prime}$ is logically equivalent to $\mathcal{O}$, i.e. $\mathcal{O}^{\prime}$ and $\mathcal{O}$ have the same models. Moreover, since $\mathcal{G}$ consists of Subset f axioms only, checking whether $\mathcal{O} \models$ subset of ( $C_{i} C_{j}$ ) can be done in polynomial time, which implies that computing $\mathcal{O}^{\prime}$ from $\mathcal{O}$ can be done in polynomial time, and that the size of $\mathcal{O}^{\prime}$ is polynomial with respect to the size of $\mathcal{O}$.

We now turn to the notion of canonical model of a completion $\mathcal{O}^{\prime}$ of an ontology $\mathcal{O}$, which is a particular interpretation for $\mathcal{O}^{\prime}$, denoted $\operatorname{can}\left(\mathcal{O}^{\prime}\right)$, defined as follows: 
- The domain of $\operatorname{can}\left(\mathcal{O}^{\prime}\right)$ contains one element $v$ for each token $v$ in $\mathcal{O}^{\prime}$, one element $e$ for each individual $e$ that is an instance of the class Experiment in $\mathcal{O}^{\prime}$, one element $s$ for each individual $s$ that is an instance of the class Sample in $\mathcal{O}^{\prime}$, and one element $e_{\mathrm{d}, \mathrm{A}, C}$ for each triple $(d, \mathrm{~A}, C)$ such that the assertion ClassAssertion ( ObjectSomeProperty ( hastinkToObject $C$ ) d ) is in $\mathcal{O}^{\prime}$.

- The extensions of the various predicates in $\operatorname{can}\left(\mathcal{O}^{\prime}\right)$ are as follows:

- For every $e$ in the domain of $\operatorname{can}\left(\mathcal{O}^{\prime}\right)$, e $\in$ Experiment ${ }^{\operatorname{can}\left(\mathcal{O}^{\prime}\right)}$ if and only if the assertion ClassAssertion ( Experiment $e$ ) is in $\mathcal{O}^{\prime}$.

- For every $\mathrm{s}$ in the domain of $\operatorname{can}\left(\mathcal{O}^{\prime}\right)$, $\mathrm{s}$ $\in$ Sample $e^{\operatorname{can}\left(\mathcal{O}^{\prime}\right)}$ if and only if the assertion ClassAssertion ( Sample $s$ ) is in $\mathcal{O}^{\prime}$.

- For every $\mathrm{d}$ and $\mathrm{v}$ in the domain of $\operatorname{can}\left(\mathcal{O}^{\prime}\right)$, and for every hasvalueFor-A, $(\mathrm{d}, \mathrm{v}) \in$ hasValueFor-A ${ }^{\operatorname{can}\left(\mathcal{O}^{\prime}\right)}$ if and only if the assertion DataPropertyAssertion ( hasValueFor-A d $\mathrm{v}$ ) is in $\mathcal{O}^{\prime}$.

- For every e and $\mathrm{s}$ in the domain of $\operatorname{can}\left(\mathcal{O}^{\prime}\right),(e, s)$ $\in$ consistsOf ${ }^{\operatorname{can}\left(\mathcal{O}^{\prime}\right)}$ if and only if the assertion objectPropertyAssertion ( consistsof e $\mathrm{s}$ ) is in $\mathcal{O}^{\prime}$.

- For every $d$ and $e_{d, \mathrm{~A}, C}$ in the domain of $\operatorname{can}\left(\mathcal{O}^{\prime}\right), \quad\left(\mathrm{d}, e_{\mathrm{d}, \mathrm{A}, C}\right) \in$ consistsOf $\mathrm{f}^{\operatorname{can}\left(\mathcal{O}^{\prime}\right)}$ and $e_{\mathrm{d}, \mathrm{A}, C} \in C^{\operatorname{can}\left(\mathcal{O}^{\prime}\right)}$ if and only if the assertion ClassAssertion ( ObjectSomeProperty ( hastinkToobject $C$ ) d ) is in $\mathcal{O}^{\prime}$.

Note that all the axioms of $\mathcal{O}^{\prime}$ are clearly satisfied by $\operatorname{can}\left(\mathcal{O}^{\prime}\right)$, and therefore $\operatorname{can}\left(\mathcal{O}^{\prime}\right)$ is a model of $\mathcal{O}^{\prime}$. This also implies that $\operatorname{can}\left(\mathcal{O}^{\prime}\right)$ is a model of $\mathcal{O}$. The following theorem immediately derives from the relationship between $\mathcal{O}^{\prime}$ and $\operatorname{can}\left(\mathcal{O}^{\prime}\right)$.

Theorem 4.1. If $\mathcal{O}$ is an ontology, and $\mathcal{O}^{\prime}$ is its completion, then for every search query $Q, d \in \operatorname{eval}_{\mathcal{O}^{\prime}}(Q)$ if and only if $\mathrm{a}$ $\in Q^{\operatorname{can}\left(\mathcal{O}^{\prime}\right)}$.

We can now present our method for processing a search query $Q$ with respect to the ontology $\mathcal{O}$ : the set of certain answers to such query is obtained by simply evaluating $Q$ over the completion $\mathcal{O}^{\prime}$ of $\mathcal{O}$. The next theorem shows that the method is correct.

Theorem 4.2. If $\mathcal{O}$ is an ontology and $Q$ is a search query, then $d \in \operatorname{cert}_{\mathcal{O}}(Q)$ if and only if $a \in \operatorname{eval}_{\mathcal{O}^{\prime}}(Q)$.

Proof. We assume that $Q$ is a positive search query. The proof can then be easily extended to capture the general form of query.

If-part We must show that $d \in \operatorname{eval}_{\mathcal{O}^{\prime}}(Q)$ implies $d \in$ $\operatorname{cert}_{\mathcal{O}}(Q)$. Suppose that $\mathrm{d} \in \operatorname{eval}_{\mathcal{O}^{\prime}}(Q)$. From theorem 4.1 we know that $d \in Q^{\operatorname{can}\left(\mathcal{O}^{\prime}\right)}$. We now prove that, for each model of $\mathcal{O}$, there is a homomorphism from $\operatorname{can}\left(\mathcal{O}^{\prime}\right)$ to such model. Let $M^{\prime}$ be a model of $\mathcal{O}$, and let $h$ be the function defined as follows:

- For every $v$ that is a token in $\mathcal{O}^{\prime}, h(v)=w$, where $w=$ $v^{M^{\prime}}$.

- For every d in Experiment ${ }^{\operatorname{can}\left(\mathcal{O}^{\prime}\right)} \cup \operatorname{Sample}^{\operatorname{can}\left(\mathcal{O}^{\prime}\right)}$, $h(\mathrm{~d})=e$ where $e=\mathrm{d}^{M^{\prime}}$.
- For every $e_{\mathrm{d}, \mathrm{A}, C}$ in $\operatorname{can}\left(\mathcal{O}^{\prime}\right), h\left(e_{\mathrm{d}, \mathrm{A}, C}\right)$ is the object in $M^{\prime}$ such that $\left(\mathrm{d}, e_{\mathrm{d}, \mathrm{A}, C}\right) \in$ hasLinkTo- $\mathrm{A}^{M^{\prime}}$.

To show that $h$ is indeed a homomorphism, we have to show that, for every class $C, f \in C^{\text {can }\left(\mathcal{O}^{\prime}\right)}$ implies $f \in C^{M^{\prime}}$, and for every binary relation $R,(f, g) \in R^{\operatorname{can}\left(\mathcal{O}^{\prime}\right)}$ implies $(f, g) \in R^{M^{\prime}}$. The only non-trivial case is the case involving objects $e_{\mathrm{d}, \mathrm{A}, C}$. Consider an object $e_{\mathrm{d}, \mathrm{A}, C}$ and observe that $\left(\mathrm{d}, e_{\mathrm{d}, \mathrm{A}, C}\right) \in$ hasLinkTo-A $\mathrm{A}^{\operatorname{can}\left(\mathcal{O}^{\prime}\right)}$, and $e_{\mathrm{d}, \mathrm{A}, C} \in C^{\operatorname{can}\left(\mathcal{O}^{\prime}\right)}$. By construction, $h\left(e_{\mathrm{d}, \mathrm{A}, C}\right)$ is the object $f$ in $M^{\prime}$ such that $(\mathrm{d}, f) \in$ hasLinkTo- ${ }^{M^{\prime}}$. Also, since the axiom ClassAssertion ( SomeObjectProperty hastinkTo-A $C$ ) d ) is in $\mathcal{O}^{\prime}$, and $M^{\prime}$ is a model of $\mathcal{O}^{\prime}$, we have that $f \in C^{M^{\prime}}$, which means that $h\left(e_{\mathrm{d}, \mathrm{A}, C}\right) \in C^{M^{\prime}}$. It follows that $h$ is indeed a homomorphism.

Now, by virtue of the property of homomorphism [31], we have that $\mathrm{d} \in Q^{\operatorname{can}\left(\mathcal{O}^{\prime}\right)}$ implies that there is a homomorphism $h^{\prime}$ from the query $Q$ to $\operatorname{can}\left(\mathcal{O}^{\prime}\right)$. By composing the functions $h^{\prime}$ and $h$, we obtain a new homomorphism from $Q$ to $M^{\prime}$, and this proves that $\mathrm{d} \in Q^{M^{\prime}}$. We have thus shown that $\mathrm{d} \in$ eval $_{\mathcal{O}^{\prime}}(Q)$ implies $\mathrm{d} \in Q^{M^{\prime}}$ for every model $M^{\prime}$ of $\mathcal{O}$, i.e. $d \in \operatorname{cert}_{\mathcal{O}}(Q)$.

Only-if-part We must show that $\mathrm{d} \notin \operatorname{eval}_{\mathcal{O}^{\prime}}(Q)$ implies $\mathrm{d} \notin \operatorname{cert}_{\mathcal{O}}(Q)$. The proof is immediate. Indeed, it is easy to verify that $\mathrm{d} \notin \operatorname{eval}_{\mathcal{O}^{\prime}}(Q)$ implies $\mathrm{d} \notin Q^{\operatorname{can}\left(\mathcal{O}^{\prime}\right)}$, and, since $\operatorname{can}\left(\mathcal{O}^{\prime}\right)$ is a model of both $\mathcal{O}^{\prime}$ and $\mathcal{O}$, this means that there is a model of $\mathcal{O}$ where d does not satisfies the query $Q$, which proves that $\mathrm{d} \notin \operatorname{cert}_{\mathcal{O}}(Q)$.

As for the computational complexity of the algorithm, the following observations hold:

- As we said before, computing $\mathcal{O}^{\prime}$ from $\mathcal{O}$ can be done in polynomial time, and the size of $\mathcal{O}^{\prime}$ is polynomial with respect to the size of $\mathcal{O}$.

- Since a search query $Q$ can be seen as a first-order query, whose basic components are tree-shaped conjunctive queries, computing the set $\operatorname{eval}_{\mathcal{O}^{\prime}}(Q)$, given $Q$ and $\mathcal{O}^{\prime}$, can be done in LOGSPACE with respect to the size of $\mathcal{O}^{\prime}$; this implies that computing the certain answers to search queries with respect to $\mathcal{O}$ can be done in polynomial time with respect to the size of both $\mathcal{O}$ and $Q$.

The next section illustrates an efficient implementation of the algorithm, based on the use of a semantic index.

\section{Practical Deployment}

In this section we give technical details of the implementation of our S.O.S. GeM solution. First, Section $5.1 \mathrm{ex}-$ plains the process of constructing the S.O.S. GeM Semantic Knowledge Base (SKB), i.e. the practical realization of the completion $\mathcal{O}^{\prime}$ of the ontology $\mathcal{O}$ discussed in Section 4. In practice, the SKB includes the concepts extracted from ENCODE metadata and their associated concepts inferred by using well-known biomedical ontologies. Then, we explain the search process for queries over ENCODE metadata (Section 5.2). Finally, we illustrate a Web-based interface (Section 5.3), dedicated to non-expert users, which provides a simple access to ontology-based query of ENCODE data and its obtained results. 


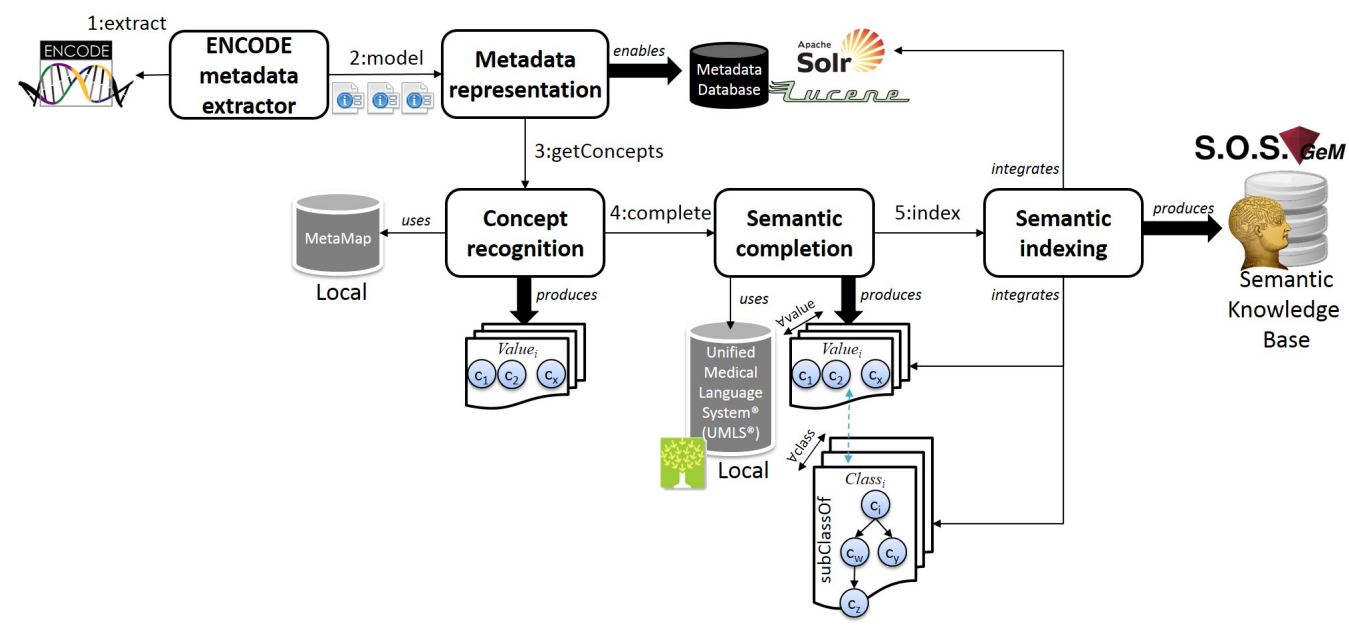

Fig. 2. Workflow to create the S.O.S. GeM Semantic Knowledge Base of ENCODE metadata.

\subsection{Semantic Knowledge Base Creation}

In building the SKB, we distinguish five different steps, which are performed sequentially in an offline process. They are depicted in Figure 2, namely ENCODE metadata extraction, metadata representation, concept recognition, semantic completion computation and the final semantic indexing.

\subsubsection{ENCODE metadata extraction}

This first step aims at producing the metadata set $M$ by extracting the freely-available ENCODE metadata. Our extractor is a Python script that crawls the ENCODE site and collects all metadata for human and mouse ENCODE data.

The crawling process is performed sequentially: our metadata extractor explores each provided URL and navigates the subdirectories that group the experiments; for each of them, the extractor retrieves a list of files and their metadata, typically named in a files.txt text file. Then, metadata associated with each listed data file in FASTQ, BAM, bigWig, bigBed, BED, broadPeak, narrowPeak or GTF data format are extracted and completed with related metadata from the ENCODE controlled vocabulary ${ }^{9}$. The final metadata are saved in a single tab delimited text file, including all the metadata attribute-value pairs (e.g. "antibody_target CTCF") for each available ENCODE data file. We adopt this data schema as this is the standard format that can be directly used in the GMQL toolkit [7].

\subsubsection{Metadata representation}

As S.O.S. GeM proposes an incremental Knowledge Base creation, the first objective is to allow a syntactic search functionality of the extracted ENCODE metadata $M$. Intuitively, S.O.S. GeM builds a database hosting the extracted metadata $M$, associating each experiment Sample data file with its set of extracted metadata attributes and values. Formally speaking, this step objective is to satisfy the matching needs of the hasValueFor-A property, representing the syntactic information $t_{S}^{\AA}(M)$ associated with each Sample $S$.

To do so, we make use of the well-known Apache Lucene/Solr 5.0 framework $^{10}$, which proves to be a feasible

9. http://hgdownload.cse.ucsc.edu/goldenPath/encodeDCC/cv.ra 10. http://lucene.apache.org/solr/ and scalable solution to tokenize and index text documents. S.O.S. GeM represents the Sample metadata using three related Lucene/Solr elements: documents, fields and nested documents. As shown in an example in Figure 3, each Sample is a Lucene/Solr document, hereinafter called ENCODE metadata document. Each document is composed of a set of attribute-value pairs describing the Sample metadata, which are encoded as Lucene/Solr fields. Following the notation in Section 4, we rename these pairs as attribute and tokens. Lucene/Solr provides different "out of the box" tokenizers and filters to normalize the text values ${ }^{11}$. In our system, we initially stick to a basic word-delimiter tokenizer.

Given that attribute-tokens fields tend to be repeated across ENCODE samples, we make use of a recent Lucene/Solr technique, named nested documents, which simulates a relational scheme having several tables and foreign keys. Thus, we place each pair in a different subdocument, called metadata pair document, which can be referenced by different Sample ENCODE metadata documents. In this way, the different pairs are stored only once, while we maintain cross-references with their associated samples which can be navigated at query time. Finally, we label each Sample with a specific field, dccAccession which corresponds to an Experiment ID), in order to quickly link the Sample to the ENCODE Experiment it belongs. Although not discussed here, we also use other fields (such as version, creation_date, etc.) for maintenance purposes.

The result of this process is a metadata database resolving the hasValueFor-A property for each Sample. This allows to retrieve the Sample metadata and the links to the corresponding ENCODE data, matching user queries.

\subsubsection{Concept recognition}

This step focuses on inspecting ENCODE metadata values and recognizing concepts that they may include from the biomedical ontologies in the Unified Medical Language System. Formally, this process analyses the text values and identifies the set of classes $c_{S}^{\mathrm{A}}(M)$ from the global ontology $\mathcal{G}$ that are in the ENCODE metadata $M$, being $\mathcal{G}$ the union of freely-available UMLS ontologies. Note that the terms "class" and "concept" are interchangeable in this paper.

11. https://wiki.apache.org/solr/AnalyzersTokenizersTokenFilters/ 


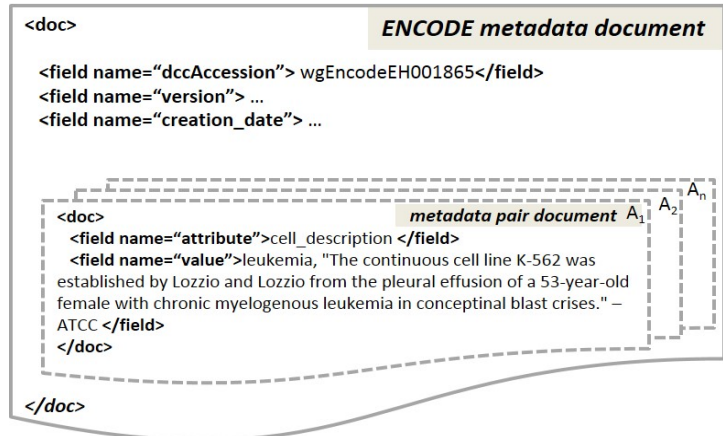

Fig. 3. A first ENCODE metadata document.

S.O.S. GeM performs this task on the basis of MetaMap [32], a tool specifically designed to discover concepts in the UMLS Metathesaurus [8] that are referred to in a given text. MetaMap relies on a knowledge intensive approach based on symbolic, natural language processing and computational linguistic techniques; its efficiency has been largely shown for this particular task. S.O.S. GeM manages a local installation of MetaMap (version 2014) to boost the performance, by processing all the information requests through the provided MetaMap Java API (release 2014), and obtain the set of UMLS concepts found in each metadata value.

It is worth recalling the integration effort provided by the UMLS Metathesaurus [8]. Current UMLS version (2014AB) includes 173 vocabularies from twenty-one languages, contributing with more than three million concepts (grouping almost twelve million atoms). The UMLS model is built under two main notions, namely concept and atom. A concept is an abstract unit of meaning, such as heart or myocardial infarction, that groups multiple (or at least one) vocabulary atoms, which are manually assessed to be synonyms in particular vocabularies. For instance, myocardial infarction groups "heart attack" in the Alcohol and Other Drug Thesaurus (AOD) and "cardiovascular stroke" in the Medical Subject Headings (MeSH). Furthermore, lexical variations of atoms (punctuation, language, etc.) are integrated and linked to their related concepts. Besides providing a curated repository of many biomedical vocabularies, UMLS includes relationships among concepts in different vocabularies, being synonyms the mappings of particular interest.

S.O.S. GeM parses each different metadata value and uses MetaMap to recognize all atoms/variations in the provided text, so that to provide as a result the UMLS concept grouping all the atom set. Since all atoms/variations under the same concept are synonyms, this allows us to minimize space and to manage a unique reference concept per recognized class. The recognized concepts per value are then stored in an enriched metadata pair document, as shown in Figure 4. In this way, we give a realization for the class objectSomeProperty ( hasLinkTo-A C ) representing the fact that each Sample is associated with a set of objects that are instances of the various classes in $c_{S}^{\mathrm{A}}(M)$.

Additional fields store the position of each concept within each metadata value, in order to highlight the semantic concept found to the user when answering user queries.

Note that we always maintain the Apache Lucene/Solr index (see Section 5.1.2) that regards the metadata value text as a sequence of syntactic tokens $t_{S}^{\mathrm{A}}(M)$. This allows an-

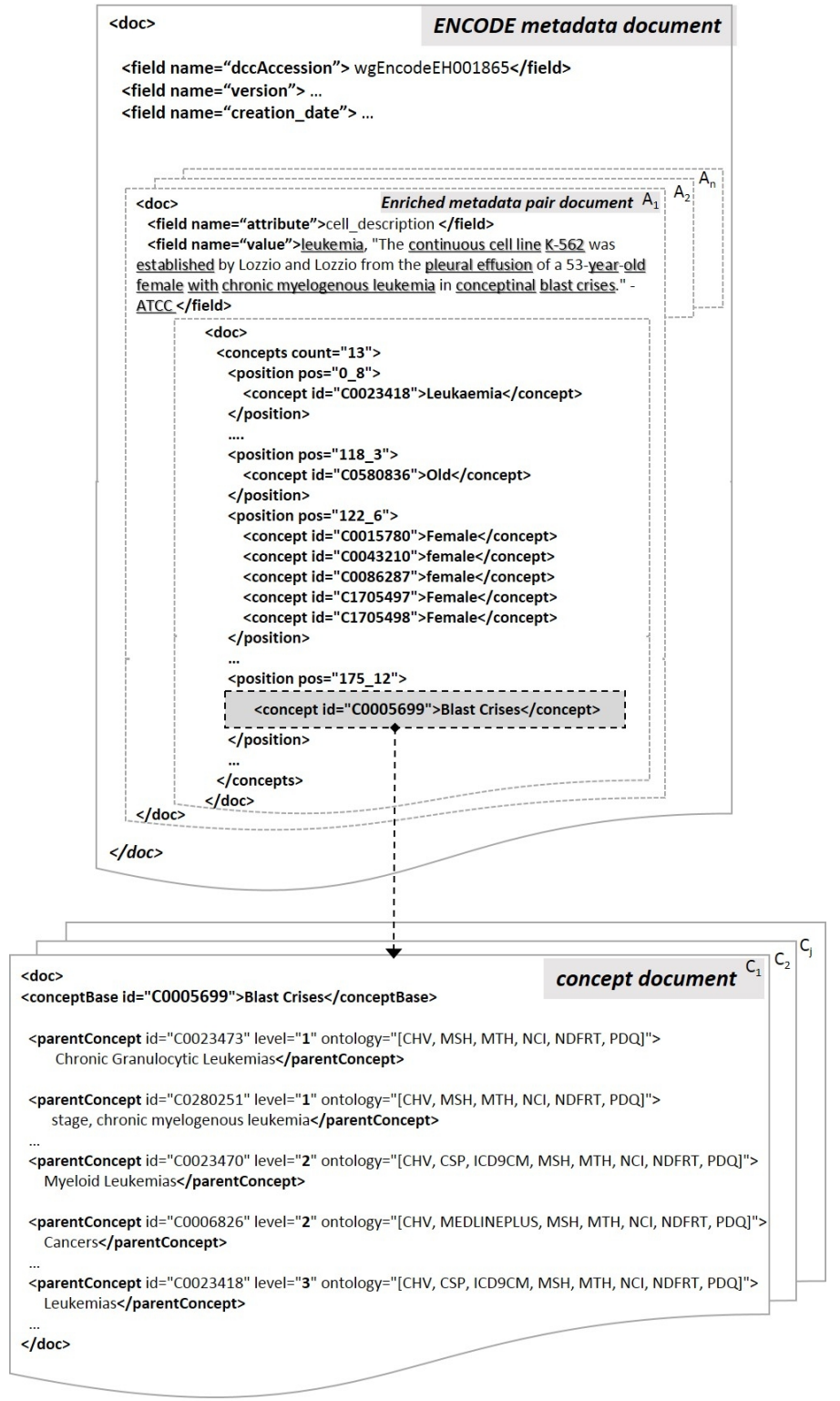

Fig. 4. Enriched ENCODE metadata and concept documents.

swering user queries both semantically (on the recognized concepts) and syntactically (on relevant syntactic tokens), as practically described in Section 5.2. Thus, even in the rare event that no concept is recognized by MetaMap (which is based on UMLS that includes more than three million concepts), user queries would be still matched syntactically.

\subsubsection{Semantic completion computation}

After the phase of concept recognition, our SKB corresponds to the ontology $\mathcal{O}$, as described in Section 4 . In the next step, we compute the completion $\mathcal{O}^{\prime}$ of $\mathcal{O}$. To do so, we need to compute the semantic completion of the various concepts, by taking care of the class assertions Subsetof.

For this purpose, S.O.S. GeM makes use of the UMLS Metathesaurus intra-source "distance 1" IS_A hierarchical relationships (immediate parents and children). Here "intra-source" denotes that these relations are not seen at the level of concepts, but at the level of atoms. That is, UMLS states the immediate Subsetof $\left(A_{i} A_{j}\right)$ relationship, where $A_{i}$ and $A_{j}$ are atoms in a given vocabulary. This means that one atom in a vocabulary can be 
navigated to get its more general concepts, or parents. For instance, in the AOD vocabulary, heart IS_A cardiovascular system, and mammal IS_A vertebrate, which formally state Subsetof ( heart cardiovascularsystem ), and Subsetof ( mammal vertebrate).

In practice, S.O.S. GeM makes use of forward chaining to materialize all the UMLS concepts that can be inferred from the recognized metadata concepts in the enriched metadata pair documents. Thus, for every single recognized concept, we retrieve all its atoms and, for every atom, we navigate its hierarchies by iteratively retrieving the subsetof relationships up to the top level concept of the specific UMLS ontology. Note that, at every step, each retrieved atom $A_{j}$ is again inspected, i.e. its associated UMLS concepts are retrieved and the process is repeated. To avoid processing each concept more than once, we maintain an ad-hoc data structure and, to make the computation efficient, we use a local installation of UMLS (ver. 2014AA on a MySQL database) to access both concept/atom data and hierarchies.

We store the semantic closure also by means of Apache Lucene/Solr documents (Figure 4). We consider each recognized UMLS concept as a document, named concept document, and we nest as many concept documents as different UMLS concepts can be inferred from it by following the Subset $O f$ relationships; we also store the number of inference steps (i.e. level) and the set of ontologies that have been used to reach each fact. For example, the fact subsetof ( primate vertebrate) is obtained in 2 steps, using $\mathrm{AOD}$ or NCI (National Cancer Institute) vocabularies, since in either vocabulary Subsetof ( primate mammal ) and Subsetof ( mammal vertebrate) hold.

\subsubsection{Semantic indexing}

The final step consists of building a semantic index for efficiently managing $\mathcal{O}^{\prime}$. More precisely, we index the enriched metadata pair documents, i.e. the ENCODE metadata set $M$ in which UMLS recognized concepts are annotated, and the concept documents, stating the Subset of-based inferred concepts. Thanks to established Apache Lucene/Solr document schema, this process is straightforward: in practice, S.O.S. GeM loads and indexes the aforementioned documents from previous processes in our Lucene/Solr backend system which, all together, constitutes the S.O.S. GeM SKB.

It is worth mentioning that, while the SKB is built once in an offline process, S.O.S. GeM allows new ENCODE samples to be easily added to the SKB, by manually loading new data samples or rerunning the S.O.S. GeM ENCODE metadata extractor and monitoring changes between versions. The updating process runs efficiently as the closure computation, and final metadata indexing sticks only to the new recognized concepts; besides adding the enriched metadata pair documents for the new data samples, only the new concept documents need to be added to the system, without affecting the rest of the SKB.

We currently do not consider sample deletions as ENCODE experiments are monotonically increasing. As for the UMLS concepts, the underlying database is typically updated just twice a year, so that S.O.S. GeM can synchronize at such given points and re-run the offline process (concept recognition, semantic completion and semantic indexing).

\subsection{Search Process}

The search process aims at enabling transparent semantic query facility to users. For example, an ENCODE metadata document including "53-year-old female" should be retrieved when the user searches for $Q$ "mammal of 53 years". Query resolution consists of query parsing and query answering.

Query parsing. S.O.S. GeM parses each query $Q$ both syntactically and semantically. First, we use the S.O.S. GeM local MetaMap instance to recognize semantic concepts, i.e. all classes $C_{i}$ contained in the provided $\beta_{i} \cdot \gamma_{i}$ of the query. Note that we follow the same configuration as for the offline SKB creation, obtaining the general UMLS concept(s) referred in the text (e.g. "mammal" and "years"), together with their associated position in the text. We take this into account to extract the non-recognized parts of the query (e.g. "of 53" in the previous example). Finally, we use a common list of stop words to filter such remaining text and extract interesting syntactic tokens $\alpha_{i}$ (e.g. "53").

Query answering. S.O.S. GeM strictly performs the evaluation of the query (Section 4.3) on the S.O.S. GeM SKB. Intuitively, S.O.S. GeM extracts (i) those samples that match the syntactic tokens, (ii) those matching the semantic concepts, and (iii) merges the results. In the first process, the syntactic tokens $\alpha_{i}$ of the query are matched against the tokens in the enriched metadata pair documents. The second process requires two complementary steps. First, we match the recognized classes $C_{i}$ in the query against the nested concept documents, i.e. we try to align each $C_{i}$ (e.g. "mammal") with the $Y$ concepts that are part of a subsetof ( $X$ $Y$ ) relationship, e.g. Subsetof (female mammal). Then, we obtain the samples from the enriched metadata pair documents which are annotated with the more specific $X$ concepts (e.g. female). Note that our query answering aims to match the user query to equally or more specific concepts, not vice versa. For instance, when users query for "mammal", S.O.S. GeM retrieves samples annotated with the concept mammal or any specific kind of mammal, e.g. mouse. Conversely, the system does not (and must not) retrieve all mammal-annotated samples when querying for mouse, as it is certainly not true that all mammals are of kind mouse.

The final merging process combines the results from both the syntactic and the semantic processes, filtering out samples satisfying the negative part of the query, $Q_{n}$, if present. To date, the S.O.S. GeM parsing process does not automatically recognize negative parts in the query, so they have to be explicitly stated. By default, S.O.S. GeM considers each provided term as a positive basic search query, so that the search query is seen as the UNION of all basic search queries. This is fully compliant with our formalization in Section 4.2 and, in practice, states that results match at least one of the provided query terms. After results are shown, in a query refinement step, S.O.S. GeM allows the user to specify which of the query terms are part of the negative query $\left(Q_{n}\right)$, and which terms are mandatory, thus grouping these latter ones in a conjunctive query inside a basic search query.

Additionally, S.O.S. GeM implements the ranking of the result set of samples. Given that the answer set could be large, we first group samples by their associated Experiment, making use of the aforementioned dccAccession property of each Sample, which unequivocally identi- 
fies the Experiment. Then, S.O.S. GeM applies a sequence of heuristics to provide a global order for this Experiment set: (i) the number of query terms, i.e. semantic concepts or syntactic tokens, matched in the experiment sample metadata (the higher the better), as not all terms might have been matched (in the default UNION interpretation, as stated in Section 4.2), (ii) the maximum number of query terms matched in a single metadata value (the higher the better), (iii) the number of different metadata attributes $A$ matched (the higher the better), (iv) the minimum number of inference steps (i.e. Subsetof relationship steps) between matching concepts (the smaller the better - the shorter the inference path, the closer is the meaning), (v) the number of inferred facts from shorter to longer paths (the higher the better - the more inferred concepts at shorter paths, the more accurate is the matching), and finally (vi) the dccAccession unique ID (taken lexicographically), in order to assure a global order.

\subsection{Web Interface: Functionalities}

S.O.S. GeM provides an intuitive Web interface ${ }^{12}$, which acts as a high-level façade of the created SKB. As stated, our main aim is to allow users to query in a natural free text, so that, after the aforementioned search process (Section 5.2), the resulting ENCODE data are found. As shown in Figure 5 , results are visualized grouped by Experiment, ordered and paginated by relevance (we paginate by the top-100 results using the heuristic in Section 5.2).

We also support the aforementioned query refinement step through the logical composition (with AND, OR or NOT operators) of the syntactic and semantic terms identified in the query text. Thus, the user can explicitly force (AND) or establish optional (OR) terms of the positive query $\left(Q_{p}\right)$, and define the negative (NOT) part of the query $\left(Q_{n}\right)$. Furthermore, we allow filtering the metadata attributes in which the query terms should be matched.

Upon user request, S.O.S. GeM shows the information supporting current results (Matching information in Figure 5); we (i) report metadata values of the samples matching the query terms, (ii) highlight matching concepts and syntactic tokens in the metadata values, and (iii) illustrate the concrete IS_A relationships (i.e. Subset of relations) that support the semantic match, together with the UMLS ontologies they belong to. Furthermore, we allow the user to select/deselect ontologies, thus showing/hiding their related facts.

Finally, for each resulting Experiment, we list the matched samples and allow downloading their extracted ENCODE metadata or available ENCODE data. To enable a bulk download, S.O.S. GeM facilitates a "Switch to File View" option, where matched samples are not grouped by experiments, so that multiple data files can be selected for downloading with their metadata files in tab-delimited attribute-value text format. This supports the direct processing of found ENCODE genomic feature data with the novel GMQL toolkit for genomic knowledge extraction [7].

\section{Evaluation}

We first provide details on the SKB size and creation performance (Section 6.1), and next a qualitative and quantitative analysis on the query performance (Section 6.2).

12. http://www.bioinformatics.deib.polimi.it/SOSGeM/

\subsection{SKB Size and Computation Time}

Table 1 shows the size of S.O.S. GeM SKB. We indexed $2,156,333$ attribute-value pairs describing the metadata of 25, 873 ENCODE samples of many different experiment types (5C, CAGE, ChIA-PET, ChIP-seq, Combined, DNAPET, DNase-DGF, DNase-seq, Exon, FAIRE-seq, Methyl, Nucleosome, Proteogenomics, Repli-chip, Repli-seq, RIP, RIP-seq, RNA-chip, RNA-PET, RNA-seq); they correspond to 710 antibody targets for 69 different human or mouse cell tissues of affected, cancer or normal cells.

We recognized 4,000 different UMLS semantic concepts, which were completed with additional 12,330 UMLS concepts; their semantic closure produced 3.5 million concept combinations over 875 different IS_A concept paths.

The SKB computation figures are shown in Table 2; they report efficient performance in space and time in all the considered processes. Our extraction process compiles a corpus of ENCODE metadata that occupies $78 \mathrm{MB}$. As expected, the MetaMap-driven concept recognition excels in performance (51 minutes), while our document-based modelling of concepts produces a total of $578 \mathrm{MB}$. The semantic completion enlarges this size up to more than 1 GB; it runs efficiently (49 minutes), taking even less time than the concept recognition phase. Finally, the last semantic indexing performs in just 28 minutes by means of Apache Lucene/Solr. Overall, the S.O.S. GeM SKB is built in slightly more than two hours; thanks to the proposed nested document modelling, its space need is reduced up to $400 \mathrm{MB}$.

\subsection{Query Performance}

S.O.S. GeM supports any type of textual queries, which can be categorized as (i) single word representing a single concept, (ii) multiple words representing a single concept, (iii) multiple words representing multiple concepts; the latter category comprises the subtype of free text queries, which can include, besides relevant words, also several words to be disregarded (e.g. verbs, adverbs, adjectives, conjunctions, etc.). For each category, we defined some biologically meaningful example queries, listed in Table 3; we used them to evaluate S.O.S. GeM performance and compare it against that of the other systems available to search for ENCODE data, i.e. the ENCODE Project Portal (ENCODE-PP) ${ }^{13}$ and the UCSC Genome Bioinformatics (UCSC-GB) ${ }^{14}$ sites.

S.O.S. GeM provides more results than the other systems (Table 3); while the other systems support just keywordbased queries, we also answer free text queries, leveraging extraction and semantic matching of query concepts. Expert evaluation of the results for the considered example queries confirmed the correctness of almost all results by S.O.S.

13. http://www.encodeproject.org/

14. http://genome.ucsc.edu/ENCODE/search.html

TABLE 1

Statistics of the S.O.S. GeM Semantic Knowledge Base

\begin{tabular}{lclclc}
\hline E & S & Metadata Pairs & C & CC & P \\
\hline \hline 3,196 & 25,873 & $2,156,333$ & 4,000 & 12,330 & 3.5 million \\
\hline
\end{tabular}

E: number of experiments; $S$ : number of samples; $C$ : number of classes; $C C$ : number of classes after completion; P: number of SubsetOf axioms 


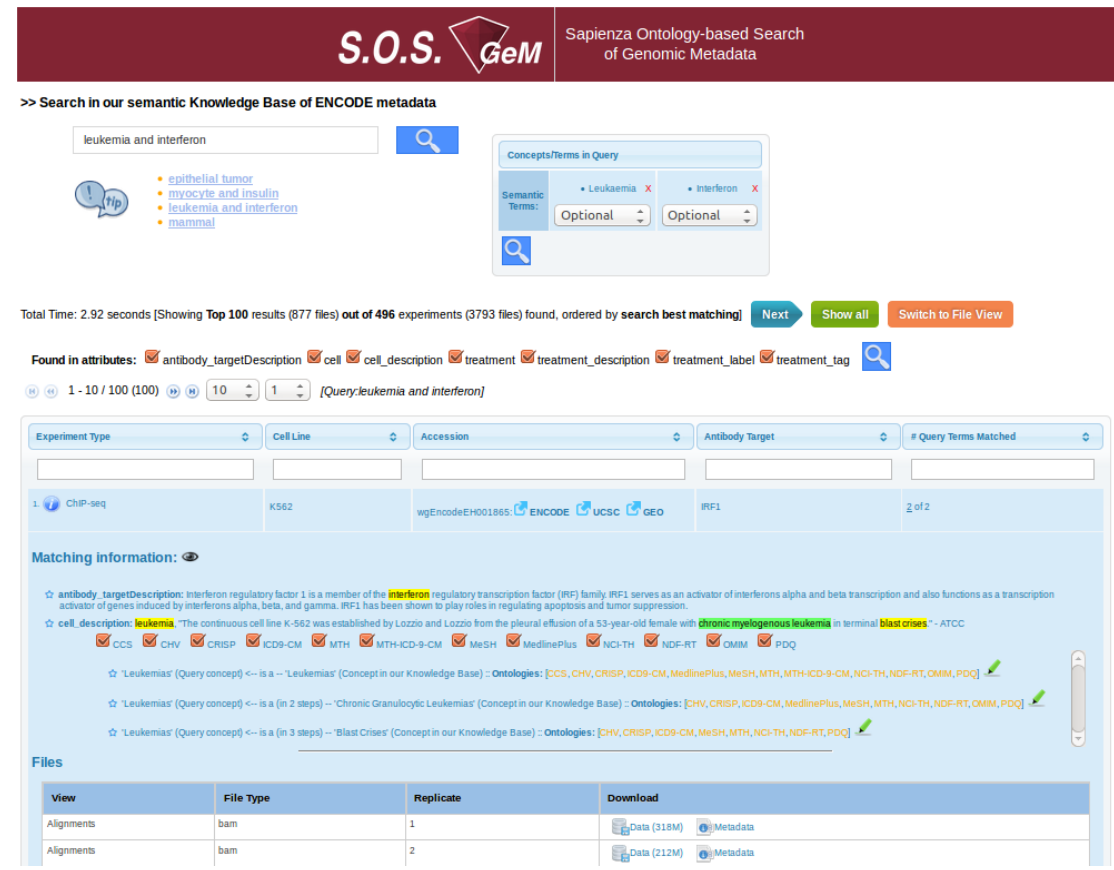

Fig. 5. S.O.S. GeM Web interface showing matching details between concepts identified in the query and in the metadata of the found experiment.

TABLE 2

Computation of the S.O.S. GeM Semantic Knowledge Base

\begin{tabular}{ccccccc}
\hline Initial & \multicolumn{2}{c}{ Concept } & \multicolumn{2}{c}{ Semantic } & \multicolumn{2}{c}{ Semantic } \\
Samples & \multicolumn{2}{c}{ Recognition } & \multicolumn{2}{c}{ Expansion } & \multicolumn{2}{c}{ Indexing } \\
Size & Time & Size & Time & Size & Time & Size \\
\hline \hline $78 \mathrm{MB}$ & $51 \mathrm{~min}$ & $578 \mathrm{MB}$ & $49 \mathrm{~min}$ & $1,137 \mathrm{MB}$ & $28 \mathrm{~min}$ & $400 \mathrm{MB}$ \\
\hline
\end{tabular}

GeM (overall correctness $98.90 \%$ ) and of most of those by UCSC-GB (overall correctness $86.73 \%$ ), while performance of ENCODE-PP on the considered queries was poor.

\subsubsection{Single word / single concept queries}

An example of this category, which shows the advantage of the semantic support in answering very specific queries, is the query "Cachectin". It is a synonym for Tumor necrosis factor (TNF), a cell signaling protein involved in systemic inflammation which is one of the cytokines (i.e. proteins) that make up the acute phase reaction. Searching for it within the ENCODE metadata using S.O.S. GeM gives as a result 20 experiments (159 sample data files), all correctly selected based on the value "TNF-alpha" of their treatment metadata attribute, which is recognized expressing the same concept of the word Cachectin; no result is found for this search using ENCODE-PP or UCSC-GB. When searching for "TNF", S.O.S. GeM finds the same results as of the "Cachectin" search, whereas ENCODE-PP and UCSC-GB retrieve only 4 experiments (42 samples) and 10 experiments (40 samples), respectively; all these results by UCSC-GB correctly match the "TNF" query, but none by ENCODE-PP does: they are wrongly retrieved just because their ENCODE-PP accession ID includes the "TNF" string. Conversely, ENCODE-PP does not retrieve the Tumor necrosis factor experiments that it includes because, apparently, its search engine does not index the experiment Treatment or Description attributes. Also the other example of this category ("estrogen") is better answered by S.O.S. GeM (with 55 experiments all correctly
TABLE 3

Types and examples of biologically meaningful queries and their results provided by S.O.S. GeM, ENCODE-PP and UCSC-GB

\begin{tabular}{|c|c|c|c|c|c|c|}
\hline \multirow{2}{*}{ Query categories and their examples } & \multicolumn{2}{|c|}{ S.O.S. GeM } & \multicolumn{2}{|c|}{ ENCODE-PP } & \multicolumn{2}{|c|}{ UCSC-GB } \\
\hline & $E$ & $S$ & $E$ & $S$ & $E$ & $S$ \\
\hline \multicolumn{7}{|l|}{ 1) single word/ single concept } \\
\hline Cachectin & 20 & 159 & - & - & - & - \\
\hline TNF & 20 & 159 & 4 & 42 & 10 & 40 \\
\hline estrogen & 55 & 449 & - & - & 35 & 104 \\
\hline \multicolumn{7}{|l|}{ 2) multiple words/ single concept } \\
\hline proto-oncogene & 58 & 500 & - & - & 26 & 74 \\
\hline peptide hormone & 73 & 465 & - & - & - & - \\
\hline white blood cell & 428 & 3,627 & - & - & - & - \\
\hline \multicolumn{7}{|l|}{ 3) multiple words / multiple concepts } \\
\hline $\mathrm{H} 1$ proto-oncogene & 6 & 42 & - & - & - & - \\
\hline leukemia interferon & 30 & 239 & - & - & 27 & 78 \\
\hline myocyte insulin & 27 & 114 & - & - & - & - \\
\hline \multicolumn{7}{|l|}{$3 b)$ free text } \\
\hline $\begin{array}{l}\text { I would like to search for CTCF } \\
\text { in human leukemia }\end{array}$ & 13 & 113 & - & - & - & - \\
\hline
\end{tabular}

E: number of experiments; S: number of samples

retrieved, while only 22 out of the 35 experiments provided by UCSC-GB were evaluated as correct by the expert, and ENCODE-PP did not get any result).

\subsubsection{Multiple word / single concept queries}

The first example of this category is the query "protooncogene". A proto-oncogene is a normal gene that can become an oncogene due to mutations or increased expression. S.O.S. GeM correctly finds 500 sample data files of 58 different experiments with various antibody targets whose encoding gene is known to be a proto-oncogene, such as Myc or c-Fos. For the same search, no results are found with ENCODE-PP, whereas UCSC-GB gives only 74 sample data files of 26 experiments, all correctly retrieved.

The second example is the query "peptide hormone". ENCODE-PP and UCSC-GB do not retrieve results for this search, although UCSC-GB finds 116 and 345 samples for 
the individual search for "peptide" and "hormone", respectively; conversely, S.O.S. GeM finds 73 experiments (465 samples), which are retrieved mainly because they regard a treatment with a peptide hormone (e.g. insulin, or activin) or a target protein related to some peptide hormone (e.g. involved in the responses of a peptide hormone). An expert reviewing these search results, through inspection of their metadata, determined that 65 these experiments are correctly retrieved; the remaining 8 are incorrectly retrieved due to the MetaMap match of the "SRF" acronym both to the "Serum Response Factor" (which is the right match in this context, but is not a peptide) and to the SomatotropinReleasing Hormone (which is a synonym of the "Growth Hormone Releasing Hormone" concept, and is a peptide).

The third example is the query "white blood cell". White blood cells are a broad category of cells present in the blood which are part of the immune system. While any biologist would be able to search for all types of cells in this category one by one, the list is long and it would be easy to forget some of them or to misspell any of their names. This search in S.O.S. GeM provides 3,627 samples of 428 experiments, which are all deemed as correct by the reviewing expert, whereas no results are found with ENCODE-PP or UCSCGB. The UMLS ontologies involved in this example are usually 7 (CHV, CRISP, FMA, LOINC, MeSH, MTH, NCI$\mathrm{TH})$. In the majority of cases the query match is found in one or two steps. In some cases the match occurs also in the antibody_targetDescription metadata attribute, and involves a higher number of semantic steps (3 or 4 ).

\subsubsection{Multiple word / multiple concept queries}

The first example is the query "H1 proto-oncogene", which adds to the term "proto-oncogene" the term "H1"; the latter one is found as a syntactic term, abbreviation of the H1hESC name of an embryonic stem cell line largely analyzed in ENCODE. In S.O.S. GeM, 42 samples of 6 experiments are found as a result of setting both terms as mandatory; all found experiments correctly regard the H1-hESC cellline and proto-oncogene related antibody targets, including c-Myc, FOSL1, MAFK and BCL11A. Both ENCODE-PP and UCSC-GB find many experiments related to $\mathrm{H} 1$, as expected, but none using the more specific query "H1 proto-oncogene".

The second example is the query "leukemia interferon". S.O.S. GeM answers with 3,793 samples of 496 experiments, related to at least one of the two concepts. Setting both terms as mandatory retrieves 239 samples of 30 experiments. Expert evaluation of the latter ones confirmed their correctness; all such experiments regard the K562 cell line, a chronic myelogenous leukemia cell line, treated with interferon alpha or gamma for different time lengths; additionally, the four top ranked experiments involve the interferon regulatory factor 1 (IRF1) as antibody target. For the same query ENCODE-PP provides no results, whereas UCSC-GB finds 78 samples of 27 experiments, all correctly retrieved.

The third example is the query "myocyte insulin". S.O.S. GeM answers with 980 samples of 154 experiments, including 114 samples of 27 experiments associated with both insulin and muscle cell concepts (since a myocyte is a muscle cell). Expert evaluation of these results proved that all of them correctly matched the query terms. Conversely, UCSCGB and ENCODE-PP provide no results for the same query.
However, UCSC-GB finds 11 samples of 4 experiments for the "insulin" query and 46 samples of 13 experiments for the "myocyte" query, whereas ENCODE-PP finds 38 experiments for the "myocyte" query, but no results for "insulin".

\subsubsection{Free text queries}

An example of such queries, which only S.O.S. GeM can answer, is "I would like to search for CTCF in human leukemia". First, S.O.S. GeM identifies the "CTCF", "human" and "leukemia" semantic terms; then, it finds 22,331 samples of 2,805 experiments whose metadata are semantically related to at least one of these concepts. When all three identified terms are set as mandatory, it finds 113 samples of 13 experiments. Expert inspection of these latter results showed that all of them correctly answer the query with appropriate ranking and can be useful to elucidate possible effects (if any) of the CTCF transcription factor in human leukemia.

\section{CONCLUSION AND FUtURE WORK}

S.O.S. GeM introduces a semantic, ontology-based approach to support the search and retrieval of ENCODE data of interest; we described in depth our solution from theoretical and practical standpoints, proving that our approach is sound and complete, and we provided a thorough evaluation. We plan to further develop and enhance S.O.S. GeM; in particular, we plan to extend it to support the search and retrieval of publicly accessible TCGA data. The public TCGA repository regards gene expressions and DNA mutations of several different cancer types from many patients (at the time of writing, 34 cancer types of more than 11,000 patients); they very well integrate with and complement the functional genomic and epigenomic data provided by ENCODE. TCGA data are also associated with metadata values of several clinical parameters characterizing the patient and biological sample from where they were obtained; thus, the S.O.S. GeM approach immediately applies to them. The effective search, retrieval and join evaluation of both ENCODE and TCGA data, using the GMQL toolkit, has a strong potential of boosting biomedical knowledge discovery.

\section{ACKNOWLEDGMENTS}

This work was supported by Austrian Science Fund (FWF): M1720-G11, and the Data-Driven Genomic Computing (GenData 2020) PRIN project (2013-2015), funded by the Italian Ministry of the University and Research. Authors thank Yuriy Vaskin for collecting the metadata of available ENCODE datasets and Massimilano Picome for his contributions in the early stage of the project.

\section{REFERENCES}

[1] E. C. Hayden, “Technology: the $\$ 1,000$ genome," Nature, vol. 507, no. 7492, pp. 294-295, 2014.

[2] C. Sheridan, "Illumina claims $\$ 1,000$ genome win," Nat. Biotechnol., vol. 32, no. 2, p. 115, 2014.

[3] 1000 Genomes Project Consortium et al., "A map of human genome variation from population-scale sequencing," Nature, vol. 467, no. 7319, pp. 1061-1073, 2010.

[4] Cancer Genome Atlas Research Network, J. N. Weinstein, E. A. Collisson, G. B. Mills, K. R. M. Shaw, B. A. Ozenberger, K. Ellrott, I. Shmulevich, C. Sander, and J. M. Stuart, "The Cancer Genome Atlas pan-cancer analysis project," Nat. Genet., vol. 45, no. 10, pp. 1113-1120, 2013 
[5] ENCODE Project Consortium et al., "An integrated encyclopedia of DNA elements in the human genome," Nature, vol. 489, no. 7414, pp. 57-74, 2012.

[6] M. C. Schatz, B. Langmead, and S. L. Salzberg, "Cloud computing and the DNA data race," Nat. Biotechnol., vol. 28, no. 7, pp. 691693, 2010.

[7] M. Masseroli, P. Pinoli, F. Venco, A. Kaitoua, V. Jalili, F. Palluzzi, H. Muller, and S. Ceri, "GenoMetric Query Language: A novel approach to large-scale genomic data management," Bioinformatics, vol. 28 , no. 7, pp. 691-693, 2015.

[8] O. Bodenreider, "The Unified Medical Language System (UMLS): Integrating biomedical terminology," Nucleic Acids Res., vol. 32, no. Database issue, pp. D267-270, 2004.

[9] R. Kontchakov, C. Lutz, D. Toman, F. Wolter, and M. Zakharyaschev, "The combined approach to query answering in $D L$ Lite," in $12^{\text {th }}$ Int. Conf. Knowledge Representation and Reasoning, 2010, pp. 247-257.

[10] M. D. Devignes, P. Franiatte, N. Messai, E. Bresso, A. Napoli, and M. Smail-Tabbone, "BioRegistry: Automatic extraction of metadata for biological database retrieval and discovery," Int. J. Metadata Semant. Ontol., vol. 5, no. 3, pp. 184-193, 2010.

[11] E. Antezana, M. Kuiper, and V. Mironov, "Biological knowledge management: the emerging role of the Semantic Web technologies," Brief. Bioinform., vol. 10, no. 4, pp. 392-407, 2009.

[12] R. Hoehndorf, M. Dumontier, and G. V. Gkoutos, "Evaluation of research in biomedical ontologies," Brief. Bioinform., vol. 14, no. 6, pp. 696-712, 2013.

[13] H. Chen, T. Yu, and J. Y. Chen, "Semantic Web meets Integrative Biology: A survey," Brief. Bioinform., vol. 14, no. 1, pp. 109-125, 2013.

[14] R. Stevens, P. Baker, S. Bechhofer, G. Ng, A. Jacoby, N. W. Paton, C. A. Goble, and A. Brass, "TAMBIS: Transparent Access to Multiple Bioinformatics Information Sources," Bioinformatics, vol. 16, no. 2, pp. 184-185, 2000.

[15] W. Xuan, M. Dai, B. Mirel, J. Song, B. Athey, S. J. Watson, and F. Meng, "Open Biomedical Ontology-based medline exploration," BMC bioinformatics, vol. 10, no. Suppl 5, p. S6, 2009.

[16] M. Ashburner, C. A. Ball, J. A. Blake, D. Botstein, H. Butler, J. M. Cherry, A. P. Davis, K. Dolinski, S. S. Dwight, J. T. Eppig et al., "Gene Ontology: tool for the unification of biology. The Gene Ontology Consortium," Nat. Genet., vol. 25, no. 1, pp. 25-29, 2000

[17] A. Doms and M. Schroeder, "GoPubMed: exploring PubMed with the Gene Ontology," Nucleic Acids Res., vol. 33, no. Suppl 2, pp. W783-W786, 2005.

[18] H.-M. Müller, E. E. Kenny, and P. W. Sternberg, “Textpresso: an ontology-based information retrieval and extraction system for biological literature," PLoS Biol., vol. 2, no. 11, p. e309, 2004.

[19] S. K. Bechhofer, R. D. Stevens, and P. W. Lord, "Gohse: Ontology driven linking of biology resources," Web Semant., vol. 4, no. 3, pp. 155-163, 2006.

[20] W. Zhu, X. Xu, X. Hu, I.-Y. Song, and R. B. Allen, "Using UMLSbased re-weighting terms as a query expansion strategy," in IEEE Int. Conf. Granular Computing, 2006, pp. 217-222.

[21] O. Thesprasith and C. Jaruskulchai, "Query expansion using medical subject headings terms in the biomedical documents," in Intelligent Information and Database Systems. Springer, 2014, pp. 93-102.

[22] M. C. Díaz-Galiano, M. T. Martín-Valdivia, and L. Ureña-López, "Query expansion with a medical ontology to improve a multimodal information retrieval system," Comput. Biol. Med., vol. 39, no. 4, pp. 396-403, 2009.

[23] J. Bhogal, A. Macfarlane, and P. Smith, "A review of ontology based query expansion," Inf. Process. Manag., vol. 43, no. 4, pp. 866-886, 2007.

[24] M. Taboada, H. Rodríguez, D. Martínez, M. Pardo, and M. J. Sobrido, "Automated semantic annotation of rare disease cases: a case study," Database, vol. 2014, p. bau045, 2014.

[25] M. Dai, N. H. Shah, W. Xuan, M. A. Musen, S. J. Watson, B. D. Athey, and F. Meng, "An efficient solution for mapping free text to ontology terms," AMIA Summit on Translational Bioinformatics, vol. 21, 2008.

[26] N. H. Shah, N. Bhatia, C. Jonquet, D. Rubin, A. P. Chiang, and M. A. Musen, "Comparison of concept recognizers for building the Open Biomedical Annotator," BMC bioinformatics, vol. 10, no. Suppl 9, p. S14, 2009.

[27] C. Jonquet, P. Lependu, S. Falconer, A. Coulet, N. F. Noy, M. A Musen, and N. H. Shah, "NCBO Resource Index: Ontology-based search and mining of biomedical resources," Web Semant., vol. 9, no. 3, pp. 316-324, 2011

[28] D. Calvanese, G. De Giacomo, D. Lembo, M. Lenzerini, and R. Rosati, "Tractable reasoning and efficient query answering in Description Logics: The DL-Lite family," J. Autom. Reason., vol. 39 no. 3, pp. 385-429, 2007.

[29] B. Motik, B. C. Grau, I. Horrocks, Z. Wu, A. Fokoue, and C. Lutz "OWL 2 Web Ontology Language Profiles (Second Edition)," 2012, W3C Recommendation 11 December 2012.

[30] B. Fazzinga, G. Gianforme, G. Gottlob, and T. Lukasiewicz, "Semantic Web search based on ontological conjunctive queries," Web Semant., vol. 9, no. 4, pp. 453-473, 2011.

[31] A. K. Chandra and D. Harel, "Structure and complexity of relational queries," J. Comput. Syst. Sci., vol. 25, no. 1, pp. 99-128, 1982.

[32] A. R. Aronson and F. M. Lang, "An overview of MetaMap: Historical perspective and recent advances," J. Am. Med. Inform. Assoc., vol. 17, no. 3, pp. 229-236, 2010.

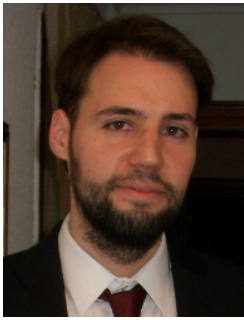

Javier D. Fernández is currently Postdoc at Vienna University of Economics and Business, Austria. He holds a PhD in Computer Science by the University of Valladolid, Spain, and the University of Chile, Chile. He is co-author of the HDT W3C Member Submission and his main interests include scalable representations and indexes for querying the Web of Data, data compression and efficient management of Big (semantic) Data.

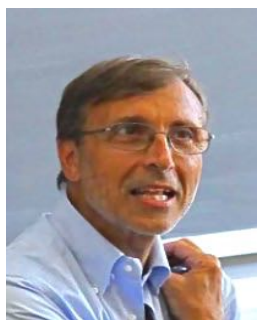

Maurizio Lenzerini is Professor at Sapienza Università di Roma, Italy, where he is leading a research group working on Database Theory, Data Management, Knowledge Representation and Automated Reasoning, and Ontology-based Data Integration. He is the author of more than 300 publications and recipient of two IBM Faculty Awards, a Fellow of the ACM since 2009 and a member of the Academia Europaea - The European Academy since 2011.

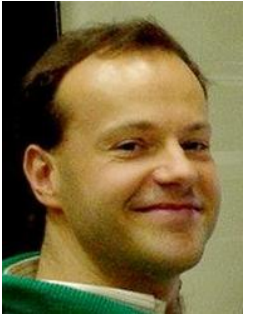

Marco Masseroli received a $\mathrm{PhD}$ in Biomedical Engineering in 1996, from Universidad de Granada, Spain. He is Associate Professor at Politecnico di Milano, Italy. His research interests are in bioinformatics and biomedical informatics, focused on distributed Internet technologies, biomolecular databases, biomedical terminologies and ontologies to effectively retrieve, manage, analyze, and semantically integrate genomic information. $\mathrm{He}$ is author of more than 170 scientific articles.

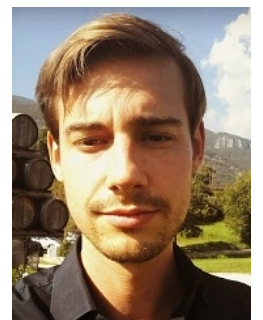

Francesco Venco is PhD candidate at Politecnico di Milano, Italy. He graduated at University of Padova with a thesis on Bayesian Network automatic learning. During the last years he worked with the group of Bioinformatics of Politecnico di Milano on techniques to manage large NGS (Next Generation Sequencing) data, in collaboration with IEO research center. His main focus is currently on efficient algorithms to manage big genomic data.

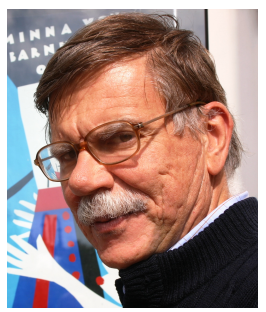

Stefano Ceri is Professor at Politecnico di Milano. His research work has been generally concerned with extending database technology to incorporate new features: distribution, objectorientation, rules, streaming data, crowd-based and genomic computing. He is currently leading the PRIN project GenData 2020. He is the recipient of the ACM-SIGMOD "Edward T. Codd Innovation Award" (2013), and an ACM Fellow and member of the Academia Europaea. 